\title{
Unsteady flow in rotating drums using laser Doppler velocimetry
}

\author{
G. Kawashima * and W.-J. Yang \\ Dept. of Mechanical Engineering and Applied Mechanics, University of Michigan, Ann Arbor, MI 48109, USA
}

\begin{abstract}
Non-destructive measurements by laser Doppler velocimetry is employed to study unsteady flow in a hollow drum filled with liquid. The drum is suddenly accelerated from rest or is suddenly decelerated from a steady rotation to rest. Pure water and glycerin-water mixtures are used as the test liquid in which polyethylenelatex particles are mixed as the light scattering tracer. The boundary layer formation, the time history of velocity, momentum and kinetic energy of the liquid, the wall-to-fluid force transfer, and the transient response time are determined. Also determined are the effects of side walls and fluid viscosity on the transient flow response. Of importance is the disclosure of Ekman layer instability near the inner radial wall of the test drum. It is actuated by the centripetal acceleration-induced buoyancy force.
\end{abstract}

\section{List of symbols}

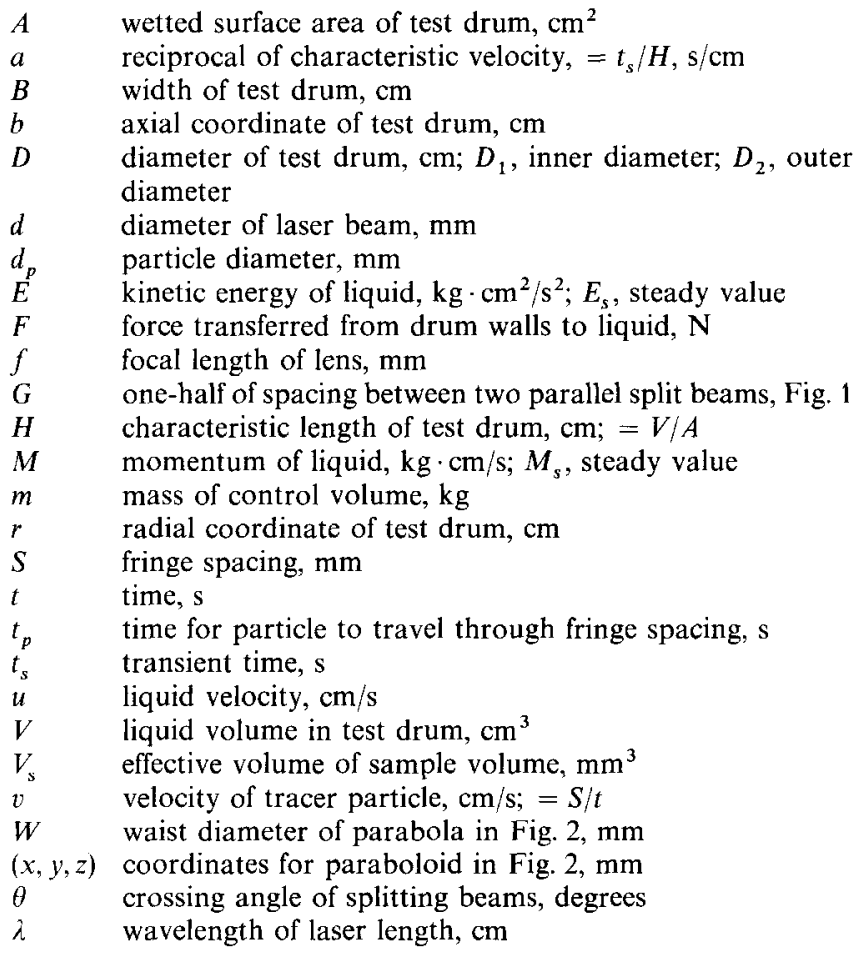

\footnotetext{
* On leave from the Dept. of Mechanical Engineering, Musashi Institute of Technology, Tokyo, Japan
}

\author{
$v \quad$ kinematic viscosity, $\mathrm{cm}^{2} / \mathrm{s}$ \\ $\varrho \quad$ liquid density, $\mathrm{kg} / \mathrm{cm}^{3}$ \\ $\phi \quad$ Doppler frequency, $1 / \mathrm{s}$ \\ Subscripts \\ $\begin{array}{ll}s & \text { at steady state } \\ 1 & \text { outer } \\ 2 & \text { inner }\end{array}$
}

\section{Introduction}

The starting process in fluid flow and boundary layer formation is commonly experienced in nature as well as industry. Its reverse is the stopping process. Typical examples are the motion which is accelerated from rest and that which is decelerated to rest.

Unsteady flow during the starting or stopping process can be studied with the aid of analytical methods which cannot be carried very much beyond the beginning of boundary layer separation. Hence, experimental investigation of such flow phenomena has been common and results are abundant. A brief survey of important studies is available (Schlichting 1968).

Recently, considerable effort has been focussed on fluid flow and heat transfer in rotating machinery which can be modelled by rotating disc systems (Owen 1984). Owen classified the rotating disc system into three categories: free disc, rotor-stator system and rotating cavity. Many rotating flows of practical importance can be considered in terms of the rotor-stator system or of the rotating cavity.

Rotating cavities can be grouped into three: sealed rotating cavity, cavity with axial through flow and cavity with radial outflow. They can be used to model the flow between co-rotating turbines or compressor discs.

Unsteady flow phenomena in rotating disc systems are less understood due to the difficulty in non-destructive measurements of velocity and pressure fields. Conventional wisdom is to insert a pitot tube or a hot-wire anemometer into the flow field. It causes a disturbance in the flow and yields 
less accurate measurements due to the weak power output from such devices.

In recent years, the application of LDV to fluid flow measurements has become popular; see, e.g., Takahei (1983) and Association for the Study of Flow Measurements (1980). It requires the presence of suitable particles in the fluid to produce enough scattered light for signal processing. Insufficient particles result in poor signal-to-noise ratios, while the diameter of seeded particles should be smaller than the fringe spacing in order to obtain high signal-to-noise ratios. Drain (1980) suggests that the optimum ratio of particle diameter to fringe spacing is 0.586 . Other references may be consulted on the selection of size (Kohashi 1974, Nakaya and Yamada 1976, Nakayama 1980) and concentration (Nakayama 1980) of tracer particles.

The present study utilizes a non-destructive means, i.e., through the use of a laser Doppler velocimeter, to monitor both the temporal and spatial variations of flow inside a rotating drum which is suddenly accelerated from rest or is suddenly decelerated from a steady rotation. The velocity, momentum, kinetic energy, force and transient response time of the fluid are investigated.

\section{Experimental apparatus and procedure}

The LDV and its signal processing system are schematically illustrated in Fig. 1. The output of the He-Ne laser light is $5 \mathrm{~m}$. When a fraction of the scattered light passing through the lens of a receiver was intercepted by an avalanche type photo diode, the scattered Doppler signals were processed by a tracker. This system was made by Cambridge Physical Science, Co., England. The resulting digital indication of Doppler frequency shift was then recorded on a printer together with the corresponding instant of measurement. An oscilloscope was installed to check whether or not the receiver was correctly catching Doppler frequency by examining the wave shape. The temperature rise due to the focusing of a laser beam depends on the wave length and the output power of the laser; in this test apparatus, the temperature rise due to the focusing of the laser light is negligible.

The power of the incident laser light is characterized by a Gaussian distribution which has the maximum intensity at the center, diminishing toward the edges of the beam. Should a lens be used to focus the beam, the resulting sample volume would take a paraboloidal form as shown in Fig. 2. The length $\Delta x, \Delta y, \Delta z$ shown in Fig. 2 are 0.15, 0.15, and $0.97 \mathrm{~mm}$, respectively.

The light-scattering particles must have the proper size and density in order to follow fluid flow. In general, the particle diameter is selected to be one half of the fringe spacing, $S$. High particle concentration causes a continuous flow of scattering particles through the sample volume. Since the angle of light scattering by each particle is fixed at the moment it enters the sample volume, the presence of multiple particles induces a composition of phase angles which

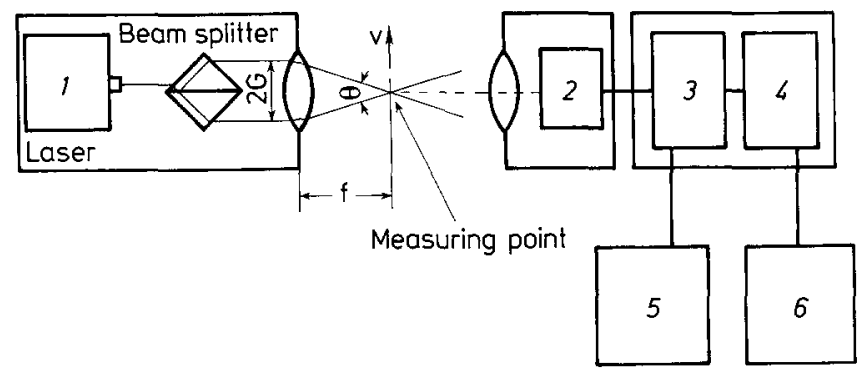

Fig. 1. A schematic of LDV and its signal processing system; 1 transmitter, 2 receiver, 3 tracker, 4 counter, 5 oscilloscope, 6 printer

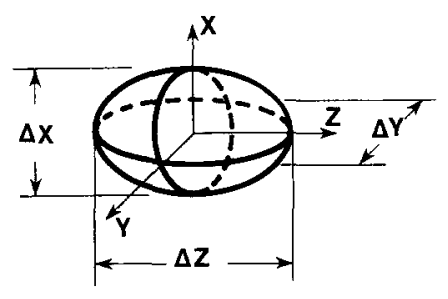

Fig. 2. Sample volume

vary with all entering and exiting particles. Hence, the desirable concentration is at one particle or less per fringe spacing.

A function generator was used to simultaneously supply signals to both the tracker and the universal counter for recording the digital response frequency in each unit. The two frequencies were then plotted on the $x$ and $y$ axes, respectively. The frequency on the tracker was corrected by the minimum square method and an equation was derived to convert the measured response frequency into flow velocity $v$.

A rotating hollow drum of $310 \mathrm{~mm}$-outside diameter and $60 \mathrm{~mm}$-width was filled with the test liquid. Under steady state with the rotating period $T r$, the actual rotational velocity $u$ and the flow velocity $v$ determined by the LDV response frequency were compared. It was disclosed that $u=v$ in the test range above $0.5 \mathrm{~cm} / \mathrm{s}$.

A highly transparent liquid is needed for LDV tests. Pure water and its mixtures with various percentages of glycerin $(25,50,65,80 \%$ by volume) were selected as the test fluids. Polyethylenelatex particles of $2.02 \cdot 10^{-3} \mathrm{~mm}$ diameter $\left(d_{p}\right)$ were mixed in the test fluid at a concentration of $0.189 \mathrm{ppm}$ (equal to the volume of one particle divided by the volume of the sample volume $V_{s}$ ). The fringe spacing was found to be $S=4.11 \cdot 10^{-3} \mathrm{~mm}$. The ratio $d_{p} / S$ of 0.491 is smaller than 0.586 (Drain 1980).

Figure 3 is a schematic of the test setup. It consisted of 1 transmitter, 2 receiver, 3 rotating hollow drum, 4 reduction gear, 5 speed controller, and 6 motor. The distance from the transmitter to the measurement point was $175 \mathrm{~mm}$. Both the distance between the measurement point and the receiver and the angle between the receiver and the laser beams were adjusted to obtain clear Doppler waves. The dimension of the test drum was varied: outer diameter 


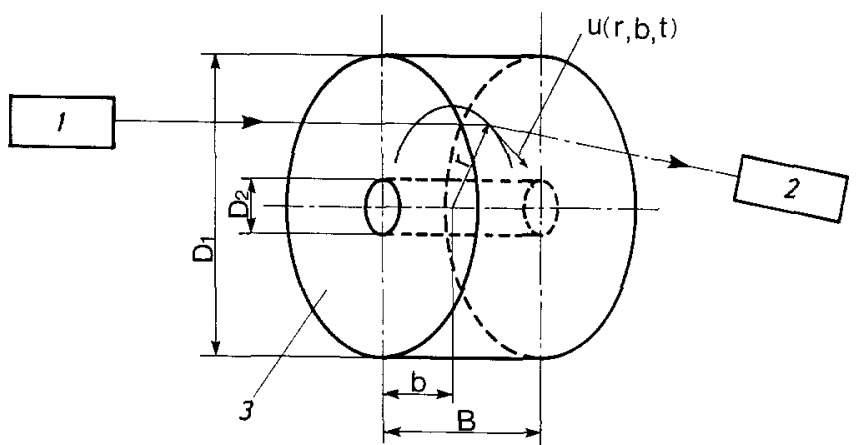

Fig. 3. A schematic of experimental apparatus

$\left(D_{1}\right)=200 \mathrm{~mm}, 310 \mathrm{~mm}$; inner diameter $\left(D_{2}\right)=60 \mathrm{~mm}$, $80 \mathrm{~mm}$; and width $(B)=30 \mathrm{~mm}, 60 \mathrm{~mm}$.

The LDV setup was a two-beam mode for one-component velocity measurements: $\theta=8.83^{\circ}, f=210 \mathrm{~mm}, G=$ $16.2 \mathrm{~mm}, \lambda=6.328 \cdot 10^{-4} \mathrm{~mm}$, and $d=0.8 \mathrm{~mm}$. Calculations yield $W=0.2115 \mathrm{~mm}$ and $V_{s}=2.282 \cdot 10^{-2} \mathrm{~mm}^{3}$, respectively. The laser light was set perpendicular to the rotating surface of the test drum. It was achieved by aligning four light points (two points by two incident lights and two points by two reflecting lights which appeared on the drum) on a straight line and at symmetrical position. In addition, the lens centers of both the transmitter and the receiver must be kept at the same height. The location of interference fringes must be on the horizontal line passing through the center of the test drum. The receiver was installed at an angle with the laser beam in order to avoid picking up the noise produced by its exposure to direct laser light.

Prior to the measurements, an oscilloscope was installed in the test setup to ascertain that the receiver intercepted the light with Doppler frequencies. The Doppler waves are characterized by smoother mountains and valleys while the noise waves have sharp peaks and bottoms like sawteeth.

Tests were conducted for both the starting and stopping processes. In both cases, the first step was to set the LDV measurement point at the measuring location in the test field. At time zero in the starting process, both the test drum and the test fluid were at rest. Then, the drum was suddenly accelerated with the rotating period $T r$ of $12.5 \mathrm{~s}$. The variation of flow velocity was monitored with time until the fluid motion achieved a steady state.

For the stopping process, the fluid flow inside the drum was initially steady at the rotating period of $12.5 \mathrm{~s}$. Then, the drum was suddenly stopped. The velocity change with time was measured until the liquid motion came to rest.

The liquid temperature was maintained constant throughout each test to avoid a change in liquid viscosity.

\section{Results and discussion}

In each test, the flow velocity $u(r, b, t)$ was measured at various locations inside the test drum. Here $r$ is the radial distance from the center of the rotating shaft, while $b$ is the axial

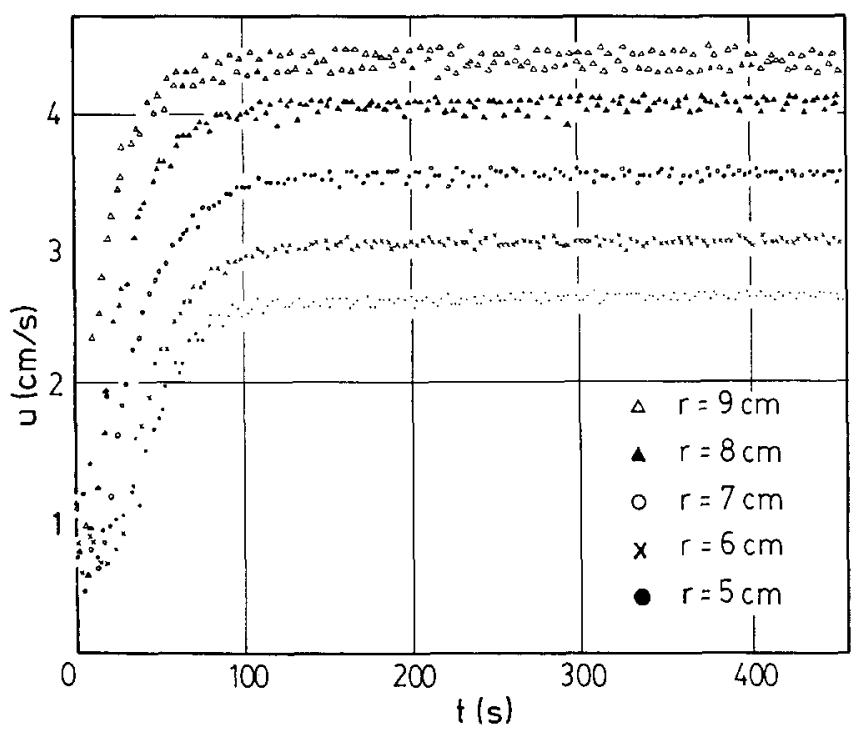

Fig. 4. Velocity response of $25 \%$ glycerin $/ 75 \%$ water mixture at $b=0$ radial cross section in test drum with $D_{1}=20 \mathrm{~cm}, D_{2}=8 \mathrm{~cm}$ and $B=6 \mathrm{~cm}$ during the starting process

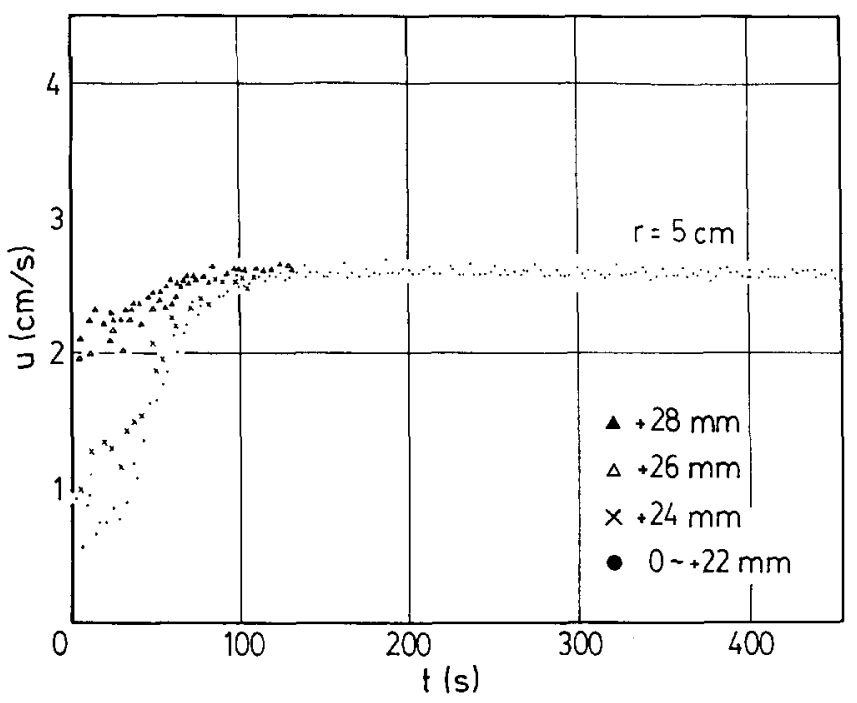

Fig. 5. Velocity response of $25 \%$ glycerin $/ 75 \%$ water mixture at $r=5 \mathrm{~cm}$ axial cross section in test drum with $D_{1}=20 \mathrm{~cm}$, $D_{2}=8 \mathrm{~cm}$ and $B=6 \mathrm{~cm}$ during the starting process

distance measured from the midplane of the test drum as seen in Fig. 3. Some typical results for the starting and stopping processes of the $25 \%$ glycerin $-75 \%$ water mixture in test drum with $D_{1}=20 \mathrm{~cm}, D_{2}=8 \mathrm{~cm}$ and $B=6 \mathrm{~cm}$ are presented in Figs. 4-11.

\subsection{Velocity time history}

Figure 4 shows the velocity time history at a radial cross section $(r, 0)$ during the starting process. For this test drum with $D_{1} / B=3.33$ and $D_{2} / B=1.33$, the flow became steady throughout the radial cross section in about 140 s. Figure 5 illustrates the velocity time history at an axial cross section 


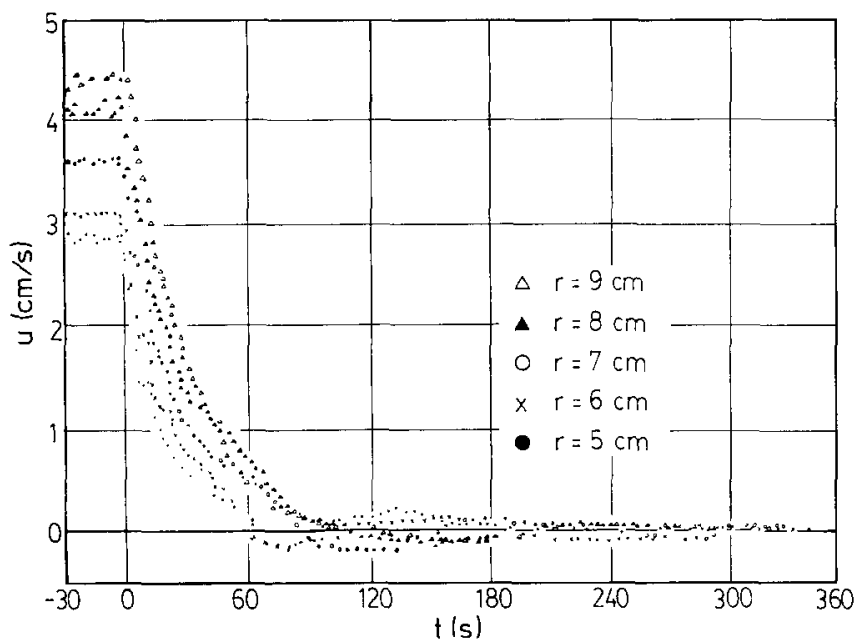

Fig. 6. Velocity response of $25 \%$ glycerin $/ 75 \%$ water mixture at $b=0$ radial cross section in test drum with $D_{1}=20 \mathrm{~cm}, D_{2}=8 \mathrm{~cm}$ and $B=6 \mathrm{~cm}$ during the starting process

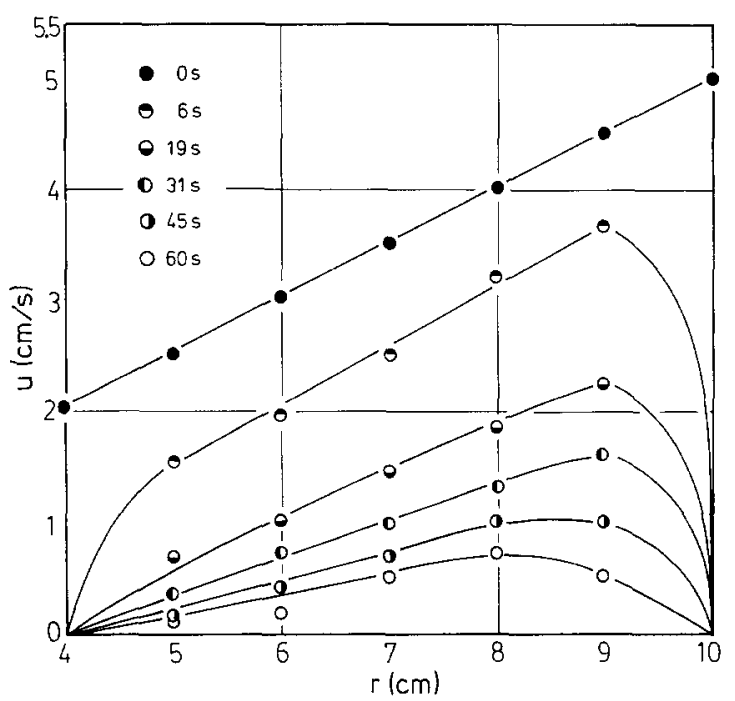

Fig. 7. Time history of $25 \%$ glycerin $/ 75 \%$ water mixture at $b=0$ radial cross section in test drum with $D_{1}=20 \mathrm{~cm}, D_{2}=8 \mathrm{~cm}$ and $B=6 \mathrm{~cm}$ during the stopping process

$(b=5 \mathrm{~cm})$. It is observed that the closer to the side wall, the faster is the response. The difference in responses along the axial cross section diminished with an increase in $r$ (not shown). Strong velocity fluctuations were detected during the initial stage near the inner radial wall as seen in Figs. 4 and 5. It is the Ekman layer instability (Greenspan 1968, Yih 1977) which was subsided as the mean velocity was increased. The centripetal acceleration gives rise to buoyancy forces which induce the instability.

In the stopping process, the velocity time history at the drum midplane $(r=0)$ is shown in Fig. 6. Steady flow was reached throughout the entire radial cross section in about $90 \mathrm{~s}$. Of special interest was the observation of the Ekman layer instability, namely the alternation of forward and reverse flows inside the boundary layer, near the inner radial

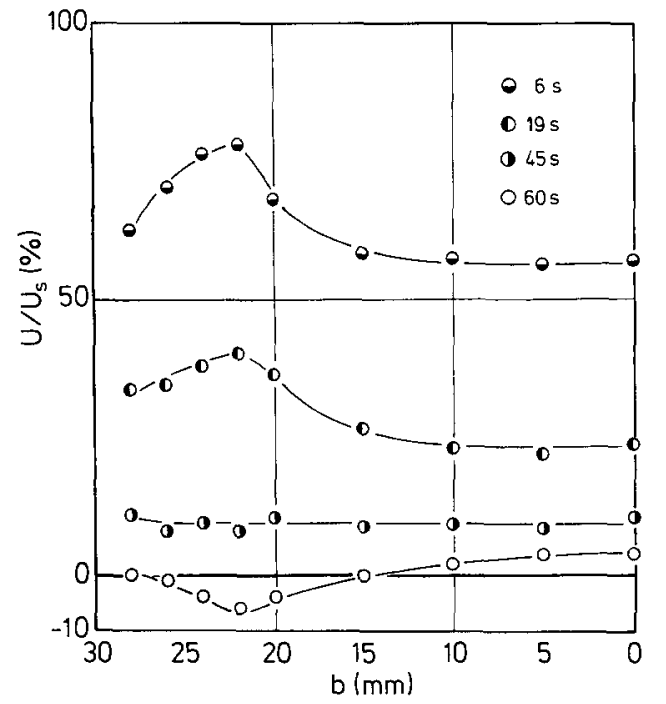

Fig. 8. Time history of $25 \%$ glycerin $/ 75 \%$ water mixture at $r=5 \mathrm{~cm}$ axial cross section in test drum with $D_{1}=20 \mathrm{~cm}$, $D_{2}=8 \mathrm{~cm}$ and $B=6 \mathrm{~cm}$ during the stopping process

surface. The instability diminished with time due to a rapid fall of the angular velocity (implying the centripetal acceleration) as will be depicted later in Fig. 12.

It was disclosed that the response time decreases with an increase in the surface area of side walls (with larger $D_{1} / B$ and smaller $D_{2} / B$ ) and the fluid viscosity.

\subsection{Time history of velocity distributions}

The time history of velocity distributions are presented in Figs. $7-10$ for the stopping and starting processes, respectively.

In the stopping process, Figs. 7 and 8 present the timewise variation of velocity distributions in the radial and axial direction, respectively. During the initial stage following the transient, the velocity profile in the radial direction diminishes linearly with time (Fig. 7), except the liquid layers near the inner and outer walls in which the Ekman layers were formed. Figure 8 depicts the action of boundary layer near the side wall at $b \geqq 20 \mathrm{~mm}$. Inside the boundary layer, an unstable velocity profile was exhibited at $r=5 \mathrm{~cm}$. A reverse flow occurred at $t=60 \mathrm{~s}$. This unstable velocity pattern, namely the Ekman layer instability, diminished with an increase in $r$. However, outside the boundary $(b<20 \mathrm{~mm})$ the velocity profile is nearly uniform (more so at larger $r$ ) at any time instant. In the case of water (not shown) the velocity decrease was slower both inside and outside the boundary layer compared with the mixture case. This is because the energy needed to induce the flow is originated from the drum walls. The energy transfer to the liquid becomes more difficult in a liquid with lower viscosity or in a drum with larger side walls (larger $\left(D_{1}-D_{2}\right) / B$ ).

In the starting process, the velocity response grew more rapid as the location was shifted radially from the inner wall 


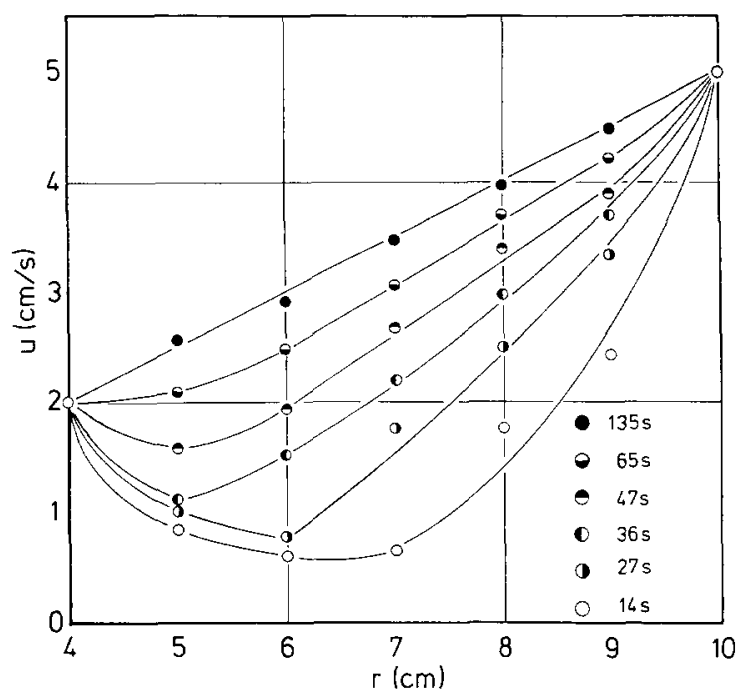

Fig. 9. Time history of $25 \%$ glycerin $/ 75 \%$ water mixture at $b=0$ radial cross section in test drum with $D_{1}=20 \mathrm{~cm}, D_{2}=8 \mathrm{~cm}$ and $B=6 \mathrm{~cm}$ during the starting process

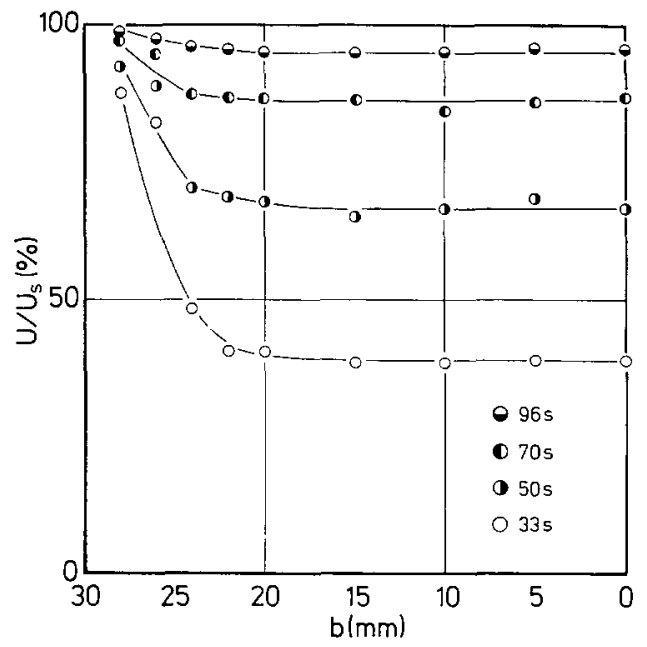

Fig. 10. Time history of $25 \%$ glycerin $/ 75 \%$ water mixture at $r=5 \mathrm{~cm}$ axial cross section in test drum with $D_{1}=20 \mathrm{~cm}$, $D_{2}=8 \mathrm{~cm}$ and $B=6 \mathrm{~cm}$ during the starting process

toward the outer wall, as seen in Fig. 9. This is also true for water, except the velocity response was slower than that in the mixture. Like in the stopping process, the boundary layer formation was observed near the side wall $(z \geqq 20 \mathrm{~mm})$, as depicted in Fig. 10. The velocity response was very rapid inside the boundary layer. The unsteadiness of velocity profiles near the inner wall is depicted in Fig. 11 at $t=33 \mathrm{~s}$. This evidences the Ekman layer instability which is gradually subsided with time.

\subsection{Angular velocity}

Figure 12 demonstrates the angular velocity response of the mixture for the stopping process. It diminishes exponentially

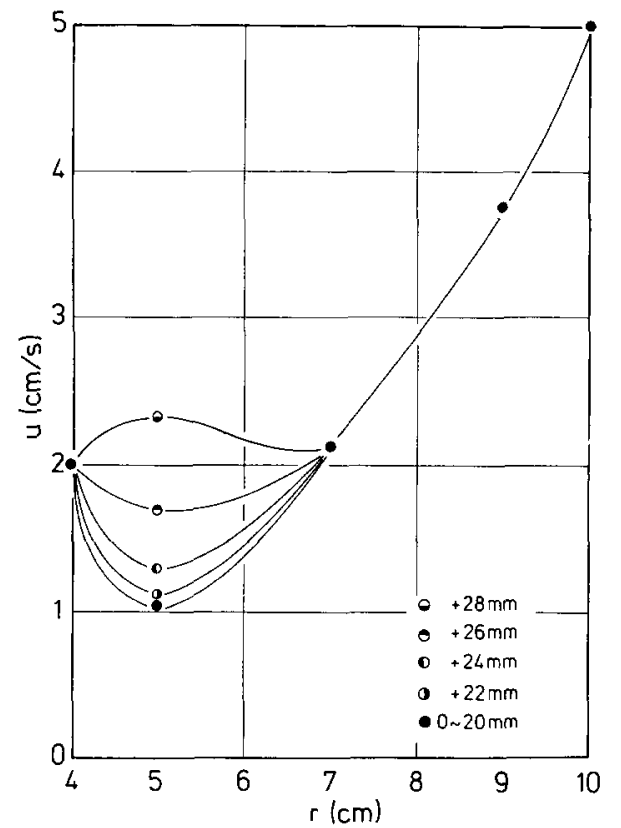

Fig. 11. Instantaneous velocity distribution of $25 \%$ glycerin $/ 75 \%$ water mixture at $b=0$ radial cross section in test drum with $D_{1}=20 \mathrm{~cm}, D_{2}=8 \mathrm{~cm}$ and $B=6 \mathrm{~cm}$ at $33 \mathrm{~s}$ after the onset of starting process

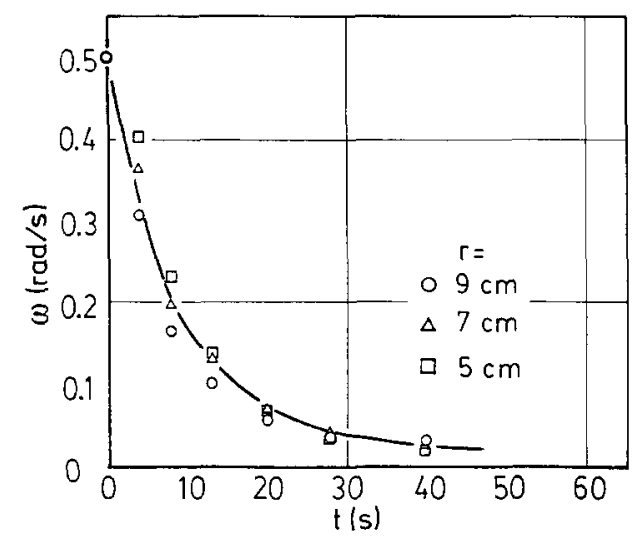

Fig. 12. Angular velocity reponse of $25 \%$ glycerin $/ 75 \%$ water mixture at $b=0$ radial cross section in test drum with $D_{1}=20 \mathrm{~cm}$, $D_{2}=8 \mathrm{~cm}$ and $B=6 \mathrm{~cm}$ during the stopping process

with time, irrespective of the radial location. A similar situation occurred in water. The angular velocity response of the starting process follows the same pattern as in Fig. 12. The quantity $r \omega^{2}$ represents the centripetal acceleration at the radial location $r$. It gives rise to the buoyancy force which can control the flow.

\subsection{Momentum and kinetic energy of liquid}

The fluid volume inside the drum was divided into a multiple of small control volumes with the spatial intervals of $\Delta r$ and $\Delta b$ in the radial and axial direction, respectively. Each control volume consisted of a ring with the rectangular cross section of $\Delta r \cdot \Delta b . \Delta r$ was selected to be $0.5 \mathrm{~cm}$ near the inner 


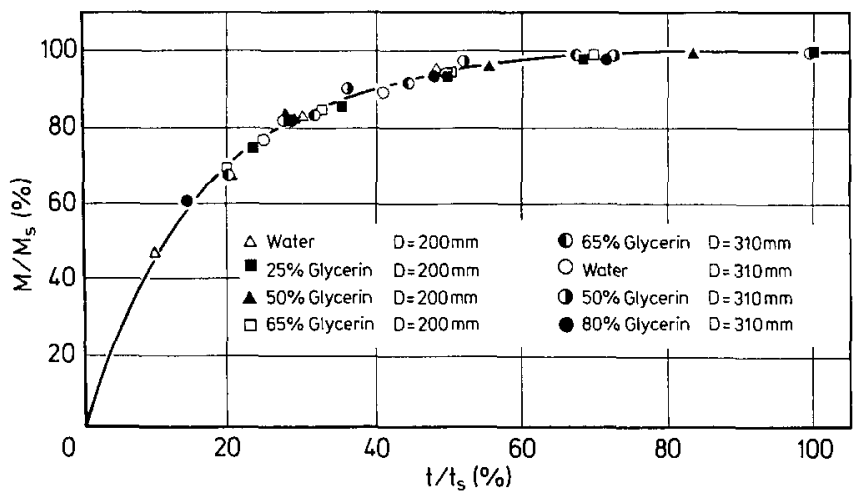

Fig. 13. Time history of liquid momentum in test drums with $b=6 \mathrm{~cm}$ during the starting process

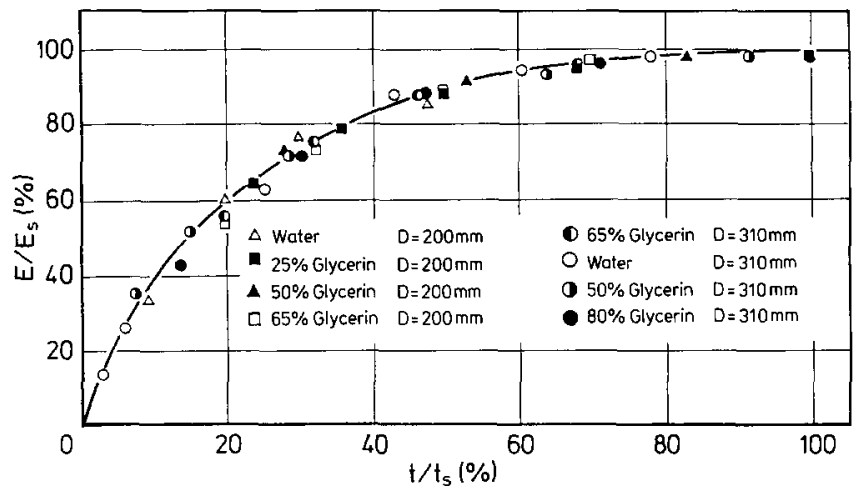

Fig. 14. Time history of liquid kinetic energy in test drums with $B=6 \mathrm{~cm}$ during the starting process

and outer radial walls and $1.0 \mathrm{~cm}$ for the radial space in between. The axial space between the midplane at $b=0$ and the side wall at $b=B / 2$ was uniformly divided at intervals $\Delta b$ of $0.5 \mathrm{~cm}$. The velocity of each ring-shaped control volume was represented by the velocity at the center of the $(\Delta r \cdot \Delta b)$ rectangle. It varied with time. Let $\Delta m$ be the mass of each control volume. $\Delta m$ is then equal to

$\Delta m=\varrho 2 \pi r \cdot 0.01 \cdot 0.005$

or

$\Delta m=\varrho 2 \pi r \cdot 0.005 \cdot 0.005$.

The fluid momentum in each control volume, $\Delta M$, is

$\Delta M=\Delta m \cdot u$.

Its integral over any time $t$ represents the instantaneous momentum $M$. Similarly, the kinetic energy of each control volume is

$\Delta E=1 / 2 \cdot \Delta m u^{2}$.

Its integral over the time becomes the instantaneous kinetic energy $E$. Both $M$ and $E$ are time-dependent.

Figures 13 and 14 illustrate $M / M_{s}$ and $E / E_{s}$, respectively. The subscript $s$ refers to the quantity at steady state, while $t_{s}$ is the time required to achieve the steady state. Both the

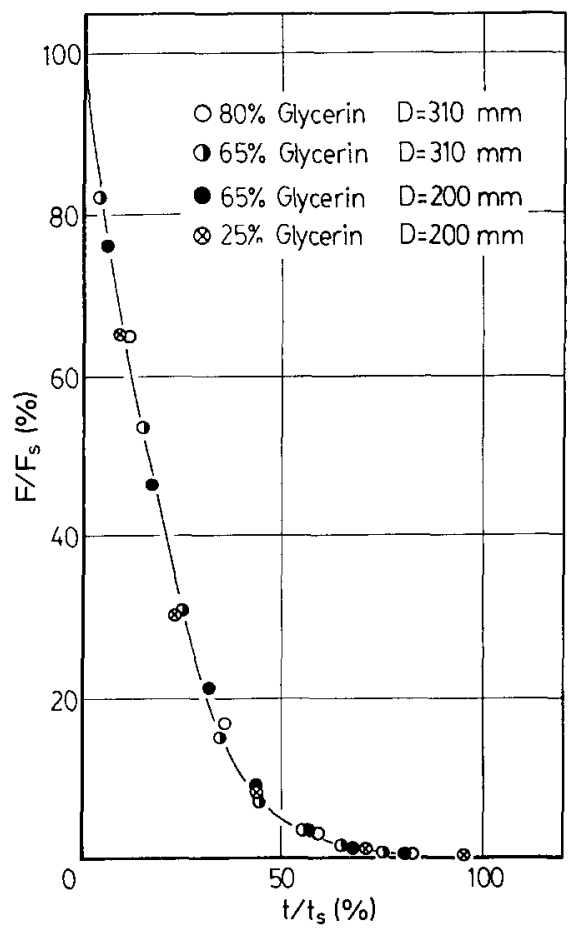

Fig. 15. Time history of wall-to-fluid force transfer in test drums with $B=6 \mathrm{~cm}$ during the starting process

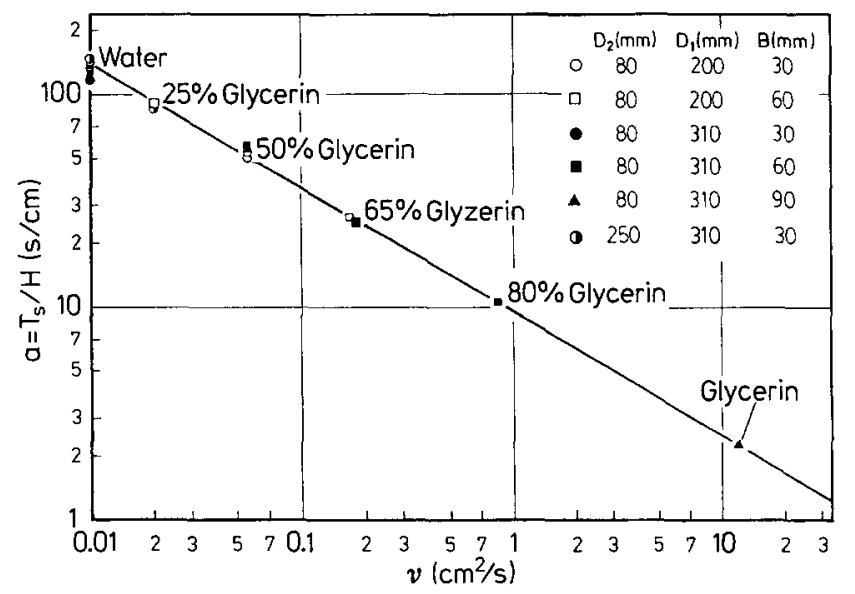

Fig. 16. Relationship between the reciprocal of characteristic velocity and kinematic viscosity

momentum and kinetic energy of the liquid responded exponentially with time.

\subsection{Wall-fluid force transmission}

Both the momentum and kinetic energy are supplied from the inner and outer radial walls and the side walls of the below drum. Therefore, the force supplied from the walls, $F$, can be expressed as the time derivative of the liquid momentum, i.e., $F=d M / d t$. $F$ was then determined by the slope of the $M-t$ plot. The result for the starting process is illustrated in Fig. 15. Eighty percent of the force was transferred to 
the fluid linearly with time in less than one-third of the transient time $t_{s}$, resulting in a very rapid response of the flow system.

\subsection{Transient response time}

The transient time $t_{s}$ is related to the volume $V$ and surface area $A$ of the hollow drum as well as the liquid property. The ratio, $H=V / A$, is the characteristic length of the drum for transport phenomena. A straight line is obtained when $a=t_{s} / H$ and the kinematic viscosity $v$ are correlated on a $\log -\log$ plot (Fig. 16). The reciprocal of $a$, namely $H / t_{\mathrm{s}}$, may be considered as the characteristic velocity of the flow system. It is logical to have a unique relationship between the characteristic flow velocity and the fluid viscosity.

\section{Conclusions}

The unsteady flow inside a rotating hollow drum is studied during the starting and stopping process by means of LDV. Pure water and glycerine-water mixtures are used as the liquid with polyethylenelatex particles as the light scattering medium. It is concluded that: (i) In both the starting and stopping processes, transient boundary layer phenomena are exhibited near the inner and outer radial walls as well as the side walls of the test drum. These boundary layers are referred to as Ekman layers. (ii) During the initial stage, one reveals flow instability (alternation of forward and reverse flows) in the Ekman layer near the inner radial wall. The centripetal acceleration gives rise to buoyancy force which induces the instability. A rapid reduction in the angular velocity with time causes a rapid decay of the instability. (iii)
The transient response time of the sealed rotating cavity reduces with an increase in the surface area of the cavity and the fluid viscosity. (iv) Both the momentum and kinetic energy of the liquid respond exponentially with time. (v) The wall-to-fluid force transfer is very rapid during the initial stage of the transient, causing a rapid flow response in both the starting and stopping processes. (vi) The characteristic velocity of the flow system is found to be uniquely related to the fluid viscosity.

\section{References}

Association for the study of flow measurements 1980: Measurements and applications of LDV, p. 10. Tokyo: Nikkan Kogyo Shinbun Sha (in Japanese)

Drain, L. E. 1980: The laser Doppler technique, p. 241. New York: Wiley

Greenspan, H. P. 1968: The theory of rotating fluids. Cambridge: Cambridge Univ. Press

Kohashi, Y. 1974: Flow measurements by lasers. J. Jpn. Soc. Mech. Eng. 77, 297-303 (in Japanese)

Nakaya, N.; Yamada, A. 1976: Measurements of flow by lasers. Res. Mach. 28, 377-383 (in Japanese)

Nakayama, Y. 1980: Techniques of flow measurements and visualization. Res. Mach. 32, 1447-1451 (in Japanese)

Owen, J. M. 1984: Fluid flow and heat transfer in rotating disc. systems. In: Heat and mass transfer in rotating machinery (eds. Metzger, D. E.; Afgan, N. H.), pp. 81-103. Washington/DC: Hemisphere

Schlichting, H. 1968: Boundary layer theory. New York: McGrawHill

Takahei, T. (ed.) 1983: The application of laser Doppler velocimetry. Tokyo: Power Comp

Yih, C.-S. 1977: Fluid mechanics. Ann Arbor: West River Press

Received July 21, 1987 